\title{
Leadership Redundancy in a Multiteam System*
}

\author{
Idar A. Johannessen $^{1}$, Philip W. McArthur ${ }^{2}$, and Jan R. Jonassen ${ }^{1}$ \\ ${ }^{1}$ Stord/Haugesund University College, Norway \\ ${ }^{2}$ Action Design, Cambridge, MA, USA
}

\begin{abstract}
Inspection, maintenance, and repair (IMR) operations of the subsea infrastructure off Norway's coast are performed from specialized vessels by multiteam systems. A case study shows how leadership is organized and practiced to coordinate interdependencies, and to cope with the risks inherent in this type of complex and tightly coupled operation. Leadership redundancy is proposed as a mechanism that can contribute to the smooth and safe functioning of a multiteam system operating in a volatile environment.
\end{abstract}

Keywords: HRO, reliability, multiteam systems, offshore operations, leadership, redundancy.

\section{Introduction}

Since complex and flexible organizational designs are used to conduct high-risk work in potentially extreme environments [5], we need to understand the leadership dynamics that enable them to be effective. There is a growing body of research and theory on multiteam systems (MTS)[6], [7] as well as on organizations that operate successfully in high-risk environments (HROs) [2][11]. Both of these bodies of literature contribute to our understanding of how to manage complexity. However, to our knowledge, these perspectives have not been integrated. We find no studies, for example, on commercial multiteam systems engaged in high-risk operations. Our research study provides an opportunity to fill that gap.

In this paper we examine how leadership is organized and executed in a multiteam system that performs inspection, maintenance, and repair on oil and gas installations on the Norwegian Continental Shelf - complex operations that entail substantial risks given the nature of the work and the extremity of the environment. We explain how both MTS Theory and High Reliability Theory deepen our understanding of the leadership of this particular organization, and how our findings contribute to both theories. We describe how leadership in this MTS is organized and exercised both to coordinate a highly interdependent operation and to cope with unexpected events that can compromise the effectiveness and safety of the operation. We propose that a

\footnotetext{
The Research Council of Norway and companies in the petromaritime industries in the Haugesund region have funded the project Managing Complexity in Petromaritime Operations at Stord/Haugesund University College. The authors are grateful for advice from Silvia Jordan, Preben H. Lindøe, Amy Edmondson, Nils M. Sortland, Lene Jørgensen, Amy Meltzer and Paul Wilson Glenn.
} 
previously unidentified mechanism, which we call leadership redundancy, can contribute to the reliability of this multiteam system. Finally, we discuss the implication of our findings for research and practice.

\section{Multiteam Systems and High-Reliability Organizations}

In their seminal paper on multiteam systems, Mathieu et al. [7] define the features of this unique organizational structure, and the factors that determine its effectiveness. A multiteam system (MTS) is a "team of teams" that works together to achieve a unifying purpose. The component teams in an MTS may come from the same company or from different companies. For example, an emergency response MTS is comprised of fire fighters, emergency medical technicians, a surgery team, and a recovery team. Each team has its own purpose, capabilities, and tasks, but they are united by the common goal of saving lives. To be considered part of an MTS, each component team must be interdependent with at least one other team on three dimensions: inputs (e.g. people, equipment, information), processes (e.g. the interaction required between teams to complete their respective tasks), and outputs (e.g. rewards, benefits, costs, task goals). The component teams may operate sequentially (where one team must complete its task before the next team can begin), in parallel, or in some combination of the two. Given that the component teams of an MTS are highly interdependent, the fundamental challenge they face is how to effectively coordinate their activities. For the purpose of this paper we focus on two critical factors that have been identified by MTS theory: leadership and shared mental models. ${ }^{1}$

Leadership in an MTS is complex. Leaders of the component teams must focus on what is happening both within their teams and between teams. They must ensure that their team's activities are aligned with the larger goal of the MTS. Leaders of the MTS as a whole must provide strategy and direction for the system, and coordinate the interdependent activities of all the component teams. In dynamic and volatile environments, MTS leaders must be mindful of changing and competing demands and be able to switch quickly from the routine to the non-routine. Consequently, MTS leaders face a dilemma. To ensure coordination within and between teams, procedures, roles, and tasks must be standardized so they can be implemented consistently. Standardization can, however, limit the teams' ability to adapt quickly to change. In comparison to leaders of single teams "the MTS leadership team will typically need to devote more of their work time ensuring system flexibility" [7].

Shared mental models help MTS members know what to expect from each other, communicate effectively, and coordinate their activities efficiently. MTS theory proposes four critical mental models for members of the multiteam system. They need a shared understanding of the purpose and task of their own team, and how it integrates with the goal of the MTS (task model). They must understand the capabilities and resources of each team in the system (team model), and how to coordinate their activities with those of the other teams (team interaction model). In addition, they must agree on how to adapt and adjust their actions to the demands of their dynamic environment (strategic mental model).

\footnotetext{
${ }^{1}$ See Mathieu et al. for a discussion of the role of IT and reward systems.
} 
Finally, MTS researchers have proposed that the process of multiteam systems, like individual teams, has two phases: a transition phase in which planning takes place, and an action phase during which work is executed [7]. Experimental studies have found that the ability of team leaders to coordinate their interdependent activities in the action phase of an MTS operation is influenced by the quality of their planning in the transition phase [6].

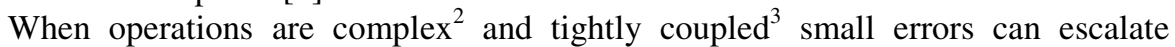
quickly and create catastrophic consequences ${ }^{4}$ [8]. Yet, some organizations that engage in high-risk work (such as nuclear power plants, submarines and aircraft carriers, and power grids), while not immune to accidents, do not experience the failure rates that one might expect [11].

Research on high reliability organizations (HROs) has identified factors that account for their success. These factors include: 1) exercising centralized control through core values, leaving decision making to the operational level $[11]$; 2) developing shared ways of thinking and acting that enable the organization to anticipate and respond to error, risk, and surprise [10]; 3) adopting hierarchical decision-making structures that encourage authority to migrate to those with the most expertise, regardless of their formal position [1]; and 4) designing multiple forms of redundancy (of workers, equipment, or capacity) into the system to increase the margin for error [9][11].

Since reliability is produced mainly through culture, it is "not bankable" [11] and must be continually regenerated. Leaders must take responsibility both for shaping, cultivating, and maintaining desired mindsets and behaviors with a long time perspective [9].

Both multiteam systems theory and high reliability theory address how organizations can succeed in turbulent, dynamic environments. Both emphasize the need for organizations to balance structure and flexibility, and both see culture (shared mental models) playing a key role to this end. MTS theory emphasizes the role that shared mental models play in coordinating interdependencies in a multiteam system, while HRT emphasizes the role that shared values and "cognitive mechanisms" (i.e., specific ways of thinking and acting) [10] play in managing surprise and error. While MTS theory identifies the leadership challenges and leadership functions in an MTS [7], HRT is more specific about how leaders and operators can think and act to ensure organizational flexibility (such as paying close attention to weak signals of impending failure, not being lulled by past success, testing assumptions publicly, and being open to diverging perspectives).

Both theories inform the interpretation of our data, and both have gaps that our research can help to address. Many studies of HROs concern organizations with multiple teams; however, the theory has not addressed the dynamics of multiteam systems as such, and the leadership challenges of this particular structure. In addition, multiteam system researchers have not conducted field studies of commercial multiteam systems comprised of teams from multiple companies that are engaged in high-risk work. MTS theory emphasizes that coordination of the MTS as a whole is a

\footnotetext{
${ }^{2}$ Many parts whose interactions are not fully predictable or even visible.

${ }^{3}$ Highly interdependent with little room for improvisation.

${ }^{4}$ This brief discussion cannot do full justice to these concepts, that are in themselves complex.
} 
critical leadership function, but it has not addressed how this coordinating role is exercised. Research on individual team leadership has proposed that effective leaders must pay attention to both task accomplishment and group maintenance [4]. MTS theory has argued that these two needs must be taken care of by the leaders of an MTS as well; however, it has not addressed how these two needs are met in practice.

\section{Design and Methodology}

Starting with the broad question about how complexity is managed in petromaritime operations, we chose to study IMR operations for three reasons: 1) they are highly complex in terms of technology, leadership, and organization; 2) they have consistently performed potentially dangerous work without major accidents; 3) they are configured similarly across the industry, which makes comparisons possible between our initial research and future research.

Our present study is based primarily on a two-week field trip following an IMR operation on one vessel. 14 semi-structured and 16 unstructured interviews were combined with some 160 hours of observation. In addition, documents were studied, and 12 interviews were conducted on shore, before and after the field trip. All interviews were transcribed and included in an NVivo database, together with the field $\log$.

Descriptions of the formal and informal organization on the vessel were created and tested for accuracy with insiders. To explore mechanisms for coping with complexity, instances where leaders faced non-trivial choices were selected from the most demanding phase of an IMR trip, the actual execution of the subsea operation.

Guided by principles of theoretical sampling [3] we sought to explore the mechanisms that permitted these operations to proceed successfully and safely. We identified non-trivial choice situations, and tracked how they were addressed and by whom. We distinguished between situations where procedures, rules, and codified knowledge seemed to provide sufficient guidance and situations in which leaders found themselves in dilemmas. We grouped and analyzed the examples of leadership dilemmas, and examined how the informants chose to deal with them. This gave us an insight into the culture and organization of leadership in the IMR context.

\section{$4 \quad$ The Research Context}

The IMR business is organized as a supply chain. The oil companies have operators on call, in our example on long-term frame contracts. Operations are performed from specialized vessels that the operators, in turn, hire from shipping companies. The IMR vessel in this research is a 110 meters long, high-tech environment with approximately 70 crewmembers.

The IMR trip is a collaboration between five companies and requires the tightly coordinated interaction of seven key individual and team roles: 1) the subsea operations team, which flies the two remotely-operated vehicles (ROVs) that perform work on the installation, 2) the tower crane operator, 3) the rigging team, 4) the main crane operator, 
5) the professional support teams, 6) the Dynamic Positioning (DP) team (which holds the vessel stationary while the subsea operation takes place), and 7) the pumping team that helps inject cleaning chemicals into the wells. The company that commissions the operation has two representatives on board; the Client Representative who is the liaison for this vessel over many trips, and the Licensee Representative, who represents the specific oil field requiring an IMR operation on this trip.

In a complex IMR operation teams must interact in mutually dependent ways. Priorities frequently change, weather conditions are unstable and most projects involve surprises. The environmental context is potentially extreme. Hannah et al. [5] define an extreme context as one "where one or more extreme events are occurring or are likely to occur that may exceed the organization's capacity to prevent and result in an extensive and intolerable magnitude of physical, psychological, or material consequences to-or in close physical or psycho-social proximity to-organization members." An IMR trip involves many potential risks for the crew, vessel (e.g. a big gas leak can sink the vessel), and the environment (e.g. pollution). The vessel is isolated at sea, often in rough weather. The different teams that carry out the operations are exposed to different levels of physical risk. The deck (where heavy objects and containers with chemicals are stored) is the most dangerous location on the vessel, especially during the mobilization and operational phases. Several activities often occur in parallel, including lifting or moving dangerous objects.

The Sub-Sea Team that flies the ROVs, and the tower crane operator who must lift and lower tools and equipment with great accuracy, operate within the closed environments of their respective control rooms. While these groups are more protected from the elements than the crews on deck, mistakes on their part can lead to catastrophic consequences.

\section{$5 \quad$ Findings}

A multiteam system can be an effective structure to harness the diverse resources necessary to carry out a complex operation, provided that the component teams are coordinated effectively [7]. Our research objective was to understand the factors that facilitate this coordination in a commercial MTS conducting high-risk work in a potentially extreme environment. Our findings in this case study are consistent with existing research and theory on multiteam systems and high-reliability organizations, and also add to those theories.

Consistent with MTS theory, an IMR operation is comprised of two distinct phases: a transition phase and an action phase. In the transition phase a series of planning and preparation processes help members of the MTS develop shared mental models about how individuals and teams are supposed to think and interact during the course of an operation. Such processes (e.g., Safe Job Analyses, Tool Box Meetings, and Task Plans) help members of the MTS to see the big picture of the operation, reinforce standard operating procedures, anticipate problems, and minimize their occurrence ${ }^{5}$. However, given the complex nature of the work and the dynamic environment in which

\footnotetext{
5 These processes are described in detail in the "Descriptive Review of IMR Operations" (forthcoming).
} 
it takes place unexpected events will occur and the system must be able to respond quickly. This has implications for the leadership structure of the operation.

While the Offshore Manager has overall responsibility for an IMR operation, once the vessel arrives over the seabed installation and the action phase starts, a transformation of the organization and leadership takes place. A hierarchical and centrally controlled multiteam system is mobilized to perform the actual work. Authority migrates from the Offshore Manager to the Shift Supervisor who is in charge of coordinating the operational MTS. He controls all operational resources, assembles all available information and decides what and how much information to distribute to the relevant parties. The leaders of the component teams, regardless of their company affiliation and their role in the hierarchy, defer to the direction of the Shift Supervisor. We also observed that the Shift Supervisor, during certain phases of the operation, would hand over control to third-party specialists. The mobilization of a latent hierarchy, and the migration of authority (with regard to sense-making and decision-making) that we observed in this MTS have been identified in previous research on high reliability organizations as factors that promote organizational reliability and effectiveness [1] [11]. Our research suggests that authority also can move upwards and laterally in the system, and that it may include several other leadership functions, such as coaching and boundary management.

During the execution of an IMR operation, the component teams of the MTS are tightly coupled. They operate within narrow timeframes, must interact in a specific sequence, and follow established procedures. Given the limited slack in the system, small errors can escalate quickly. Putting the operation on hold is a fallback option, which does get used, but which can be costly. While some component teams may experience idle time (e.g., ROV pilots waiting for equipment to be ready), the Shift Supervisor is seldom able to leave his post. He must process a continuous flow of information, and focus almost solely on coordinating the tasks of the interdependent teams, with little time left to attend to other leadership functions, such as "group maintenance" [4] of the MTS as a whole. When problems arise in the MTS (either within or between the component teams), which the team leaders are not able to manage, the Shift Supervisor is in a difficult bind.

For example, in one situation an inexperienced Deck Foreman had difficulty with a new crewmember (a rigger). The rigger was behaving erratically, seemed unaware of the potential hazards on deck, and was unresponsive to feedback. The Deck Foreman approached the Shift Supervisor for help and asked him to intervene directly. The Shift Supervisor faced a dilemma: If he intervened, he would take his attention away from coordinating the highly complex and interdependent flow of the operation. If he did not intervene, there was a risk that the rigger's behavior could cause serious problems that would jeopardize the operation and the safety of the vessel. We observed several situations in which disturbances in the MTS could create this type of dilemma for the Shift Supervisor. In some cases, as the following three examples illustrate, tensions within the MTS were managed not by the Shift Supervisor or by component team leaders, but by leaders on the vessel who were not directly engaged in executing the IMR operation.

1. In one situation there was an oil leak in one of the ROV manipulators, and the vehicle was brought to the surface for repairs. While the recovery was underway, 
several people gathered in the ROV control room, including the Client Representative. The Client Representative initiated a discussion about why the accident happened and how it could have been prevented. The Offshore Manager, who had just arrived on the scene, saw the Client Representative's intervention as inappropriate and potentially distracting for the team and for the Shift Supervisor, who was in his control room nearby. He intervened to move the discussion away from the ROV control room. The Offshore Manager in this case fills a leadership function of guarding the boundary of a team, strictly speaking under the command of the senior ROV supervisor and ultimately the Shift Supervisor. Tensions are relieved and the team can focus back on immediate damage control, while the learning discussion is moved to a space where it is more likely to succeed.

2. While informal cooperation and communication takes place regularly between all the stakeholders, difficulties arise when the Client Representative (or the License Representative) asks the Shift Supervisor to perform duties that may go beyond contractual obligations or to engage in tasks that prevent the Shift Supervisor from devoting full attention to the operation. For example, a License Representative requested that the Shift Supervisor take over responsibility for a pumping operation. The Offshore Manager, whose expertise includes knowledge of contractual matters, overheard the conversation and pointed out to the License that this was the duty of the Third Party, hired directly by the License on a different contract. Again, the Offshore Manager handles boundary management and prevents the Shift Supervisor from going beyond his competencies and potentially harming the operation.

3. In a third example, the Medic noticed that the Deck Foreman was having difficulty with his team and not delegating tasks effectively. The Deck Foreman lost oversight over activities on deck, which could jeopardize effectiveness and safety. In his role, the Medic has both the mandate and the time to address health, safety, and environment (HSE) matters on-board and make ad hoc interventions. When the Deck Foreman was off-duty, the Medic asked to speak with him and offered his feedback. In this example, a non-leader performs coaching, which would enable the Deck Foreman to be more effective in the future.

Our research findings suggest that the demands of a multiteam system performing high-risk work in a potentially extreme environment require that authority be centralized in the role of a coordinator. Under stress the coordinator will feel compelled to prioritize task coordination over other leadership functions (e.g., coaching, conflict management, and boundary management) [4]. When frictions occur in the MTS, which need immediate attention, the coordinator is in a bind. For the MTS to function effectively, other leaders who are not directly engaged in the execution of the operation step in to help. We refer to this availability of additional leadership resources as leadership redundancy.

\section{Implications}

Our findings contribute to an understanding of the challenges of an MTS in two critical areas: 1) the role that redundancies play in the effectiveness of complex 
operations; and 2) how leadership roles in an MTS can be implemented to enable effective coordination of the operation, while also attending to the inter-group maintenance of the MTS as a whole. Both findings contribute to our understanding of a core paradox identified by both MTS theory and HRT: the need for complex organizations to be both structured and flexible.

A limit of our study is that our findings are based on data from one case study. More research is underway to test our hypothesis about leadership redundancy. We need to understand the factors, other than capacity, that influence the effective utilization of leadership redundancy. We need to know how shared understandings of the appropriate use of leadership redundancy (how, when, who) are shaped. For example, not all deck foremen might welcome being coached by a medic on leadership issues. Leadership redundancy may add complexity and stress as well as relieve it, particularly if members of the multi-team system do not have a shared mental model about its execution.

\section{References}

1. Bigley, G.A., Roberts, K.H.: The Incident Command System: High-Reliability Organizing for Complex and Volatile Task Environments. Academy of Management Journal 44, 1281-1299 (2001)

2. Bourrier, M.: Das Vermächtnis der High Reliability Theory. In: Weyer, J., SchulzSchaeffer, I. (eds.) Management komplexer Systeme - Konzepte für die Bewältigung von Intransparenz, Unsicherheit und Chaos, Oldeburg, München (2009)

3. Corbin, J.M., Strauss, A.L.: Basics of qualitative research: Techniques and procedures for developing grounded theory, 3rd edn. Sage, Thousand Oaks (2008)

4. Hackman, J.R., Walton, R.E.: Leading groups in organizations. In: Goodman, P.S.A. (ed.) Designing Effective Work Groups. Jossey-Bass, San Fransisco (1986)

5. Hannah, S.T., Uhl-Bien, M., Avolio, B.J., et al.: A Framework for Examining Leadership in Extreme Contexts. Leadership Quarterly 20, 897-919 (2009)

6. Marks, M.A., DeChurch, L.A., Mathieu, J.E., et al.: Teamwork in Multiteam Systems. J. Appl. Psychol. 90, 964-971 (2005)

7. Mathieu, J.E., Marks, M.A., Zaccaro, S.J.: Multi-team systems. In: Anderson, N., Ones, D., Sinangil, H.K., et al. (eds.) Handbook of Industrial, Work and Organizational Psychology, vol. 2. Sage, London (2001)

8. Perrow, C.: Normal accidents: Living with high-risk technologies. Basic Books, New York (1984)

9. Roberts, K.H.: Some Characteristics of One Type of High Reliability Organization. Organization Science 1, 160-176 (1990)

10. Weick, K.E., Sutcliffe, K.M., Obstfeld, D.: Organizing for high reliability: processes of collective mindfulness. In: Staw, B.M., Sutton, R.I. (eds.) Research in Organizational Behavior, vol. 21, pp. 81-123. Elsevier Science/JAI Press (1999)

11. Weick, K.E., Sutcliffe, K.M.: Managing the unexpected, 2nd edn. Jossey-Bass, San Francisco (2007) 